\title{
Primer Registro de Catagonium brevicaudatum (Catagoniaceae) para la Argentina
}

\author{
First RECORD OF CATAGONIUM BREVICAUDATUM (CATAGONIACEAE) FOR \\ Argentina
}

1. Facultad de Ciencias Naturales e IML, Universidad Nacional de Tucumán, Miguel Lillo 205, (4000) San Miguel de Tucumán, Tucumán, Argentina.

2. Unidad Ejecutora Lillo,

(CONICET- Fundación Miguel Lillo), Miguel Lillo 251, (4000) San Migue de Tucumán, Tucumán, Argentina.

*t_colotti@ hotmail.com

Citar este artículo

COLOTTI, M. T. \& G. M. SUÁREZ. 2019. Primer registro de Catagonium brevicaudatum (Catagoniaceae) para la Argentina. Bol. Soc. Argent. Bot. 54: 13-16.

DOI: http://dx.doi. org/10.31055/1851.2372.v54. n1.23575

\author{
Maria T. Colotti ${ }^{*}$ (iD y Guillermo M. Suárez ${ }^{1,2}$ iD
}

\section{SUMMARY}

Background and aims: The genus Catagonium includes two species in Argentina: C. nitens y $C$. nitidum. The aim of this study was recorded Catagonium brevicaudatum Müll. Hal. ex Broth. for Argentina in the National Park Campo de los Alisos, Tucumán.

M\&M: The specimens were analyzed morphologically using conventional techniques for bryophytes and mounted in Hoyer's solution.

Results: It is distinguished from the others species of the genus by foliar characters such as leaves ovate to ovate-oblong, strongly concave, auriculate, and an abruptly piliferous, recurved apex.

Conclusions: $C$. brevicaudatum is described and illustrated, and the differences between this taxon and the other species present in Argentina are shown in a key.

\section{KeYwords}

Argentina, Bryophyta, Catagoniaceae, new record, taxonomy.

\section{RESUMEN}

Introducción y objetivos: El género Catagonium incluye dos especies en Argentina: $C$. nitens y $C$. nitidum. El objetivo de este trabajo fue registrar Catagonium brevicaudatum Müll. Hal. ex Broth. para la Argentina en el Parque Nacional Campo de los Alisos, Tucumán.

M\&M: Los especímenes fueron analizados morfológicamente mediante técnicas convencionales para briofitas y montadas en solución de Hoyer.

Resultados: La especie se distingue sobre la base de caracteres foliares como la forma de las hojas ovadas a ovado-oblongas, fuertemente cóncavas, base auriculada y ápice abruptamente pilífero y recurvado.

Conclusiones: $C$. brevicaudatum se describe e ilustra, y las diferencias entre este taxón y las otras especies del género representadas en el país se presentan en una clave.

Palabras clave

Argentina, Bryophyta, Catagoniaceae, nuevo registro, taxonomía.

\section{INTRODUCCIÓN}

El género Catagonium Müll. Hal. ex Broth. comprende musgos pleurocárpicos que se caracterizan por las plantas con hojas complanadas, dispuestas en dos hileras, ocasionalmente juláceas, las hojas son generalmente cóncavas, asimétricamente conduplicadas y los rizoides papilosos, entre otros caracteres. Es un género pequeño que incluye solo cuatro especies en el hemisferio sur distribuidas por las regiones montañosas de los trópicos y en los bosques Andino-patagónicos del sur de Chile y Argentina. Para el neotrópico dos especies han sido registradas: $C$. brevicaudatum y $C$. emarginatum. Las diferencias entre éstas se basan principalmente en el ápice de la hoja vegetativa (Lin, 1984; Gradstein et al., 2001). En la Argentina el género se conocía solamente en el extremo sur del país (Neuquén, Río Negro,
Recibido: 1 Octubre 2018

Aceptado: 21 Noviembre 2018

Publicado: 28 Marzo 2019

Editor: Gabriel Bernardello

ISSN versión impresa 0373-580X ISSN versión on-line 1851-2372 
Chubut, Tierra del Fuego e Islas Malvinas), con dos especies: C. nitens (Brid.) Cardot y C. nitidum (Hook. f. \& Wilson) Broth. (Lin, 1984; Matteri, 2003).

En esta contribución citamos por primera vez para la Argentina a C. brevicaudatum. Esta especie fue encontrada por encima de los $1000 \mathrm{~m}$ entre la vegetación característica de las Yungas del Parque Nacional Campo de los Alisos (NOA), donde abundan laureles [Ocotea porphyria (Griseb.) van der Werff] y nogales [Juglans australis Griseb.] entre otros ejemplares representativos de la Selva (Colotti et al., 2017, 2018). Incluimos una descripción, ilustración (Fig. 1), breves observaciones sobre el material estudiado, y una clave para la identificación de las especies de Catagonium presentes en la Argentina.

\section{Materiales y Métodos}

El estudio se basa en ejemplares de colectas propias realizadas en el Parque Nacional Campo de los Alisos y en la revisión de bibliografía ya publicada (Churchill \& Linares, 1995; Gradstein et al., 2001; Lin, 1984; Matteri, 2003; Sharp et al., 1994). Asimismo, se consultaron descripciones originales y ejemplares depositados en herbarios (BM, LIL, MO). Las muestras fueron procesadas de acuerdo a técnicas convencionales para briofitas (Frahm, 2003).

\section{Clave de las especies de Catagonium presentes en la Argentina.}

A. Plantas con tallos y ramas juláceas, hojas cóncavas, raramente asimétricamente conduplicadas; ápice abruptamente largo cuspidado y distalmente recto. .......... C. nitidum

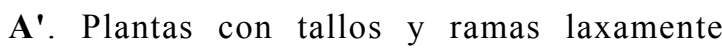
complanadas, hojas cóncavas, asimétricamente conduplicadas; ápice de forma diversa, curvado o recto.

B. Plantas de tamaño variable hojas ovadotriangular, ovado-oblongas a lanceoladas, mucronadas, acuminadas a cuspidadas; truncadas en la inserción, ocasionalmente con aurículas. ...

C. nitens

B'. Plantas delicadas, pequeñas a medianas, hojas ovadas a ovado-oblongas, abruptamente pilíferas y recurvadas, levemente auriculadas.

C. brevicaudatum

\section{Resultados}

\section{Tratamiento taxonómico}

Catagonium brevicaudatum Müll. Hal. ex Broth. in Nat. Pfl. 1(3): 1088. 1908. Fig. 1

Plantas pequeñas a medianas, verde amarillentas a doradas en tapices laxos a densos. Tallos postrados a erecto ascendentes, irregularmente ramificados, laxamente foliados, en sección transversal región cortical de 1-2 (-3) filas de células pequeñas de paredes gruesas, las internas más grandes con paredes delgadas, sin cordón central; rizoides papilosos, pardo-oscuros, en manojos laxos a lo largo de los tallos; pseudoparafilios ausentes. Pelos axilares con 1-2 células basales pardas, cortorectangulares, de paredes delgadas, lisas, de 5,0-6,25 x 7,5-12,5 $\mu \mathrm{m}$ y 2-3 células distales hialinas, largorectangulares, de paredes delgadas, lisas, de 8,75$10,0 \times 25-47,5 \mu \mathrm{m}$. Hojas dísticas, complanadas, oblongas u ovado- oblongas, de 0,6-1,2 x 0,3-0,5 $\mathrm{mm}$, conduplicadas, profundamente cóncavas; ápice obtuso redondeado abruptamente pilífero, apículo recto a flexuoso, base ligeramente auriculada; margen plano, entero; costa generalmente ausente, excepcionalmente corta y doble; células mediales linear- vermiculares, de 57,5-87,5 x 3,75-6,25 $\mu \mathrm{m}$, de paredes firmes, lisas, células basales flexuosas, irregulares, más cortas en las aurículas e inserción de la hojas, de 25,0-37,5 x 5,0-8,75 $\mu \mathrm{m}$; región alar indiferenciada. Esporofitos no observados en el material estudiado.

Distribución. Brasil, Bolivia, Colombia, Costa Rica, Ecuador, Guatemala, Jamaica, México, Perú y Venezuela (Bowers, 1994; Churchill \& Linares, 1995; Lin, 1984). En Argentina se la registra por primera vez y se distribuye en el noroeste del país.

Comentarios. Durante la revisión del material solicitado a los herbarios (LIL, BM, MO) observamos un sintipo de $C$. nitidum recolectado por Menzier en Staten Island (18-BM). Caracteres distintivos de esta especie son las plantas con tallos y ramas juláceas y hojas dispuestas en forma imbricada. Sin embargo, el ejemplar difiere en que presenta plantas con tallos y ramas foliadocomplanadas y hojas conduplicadas, truncadas en la inserción, además el ápice es largo cuspidado, 
M. T. Colotti y G. M. Suárez - Catagonium brevicaudatum en Argentina

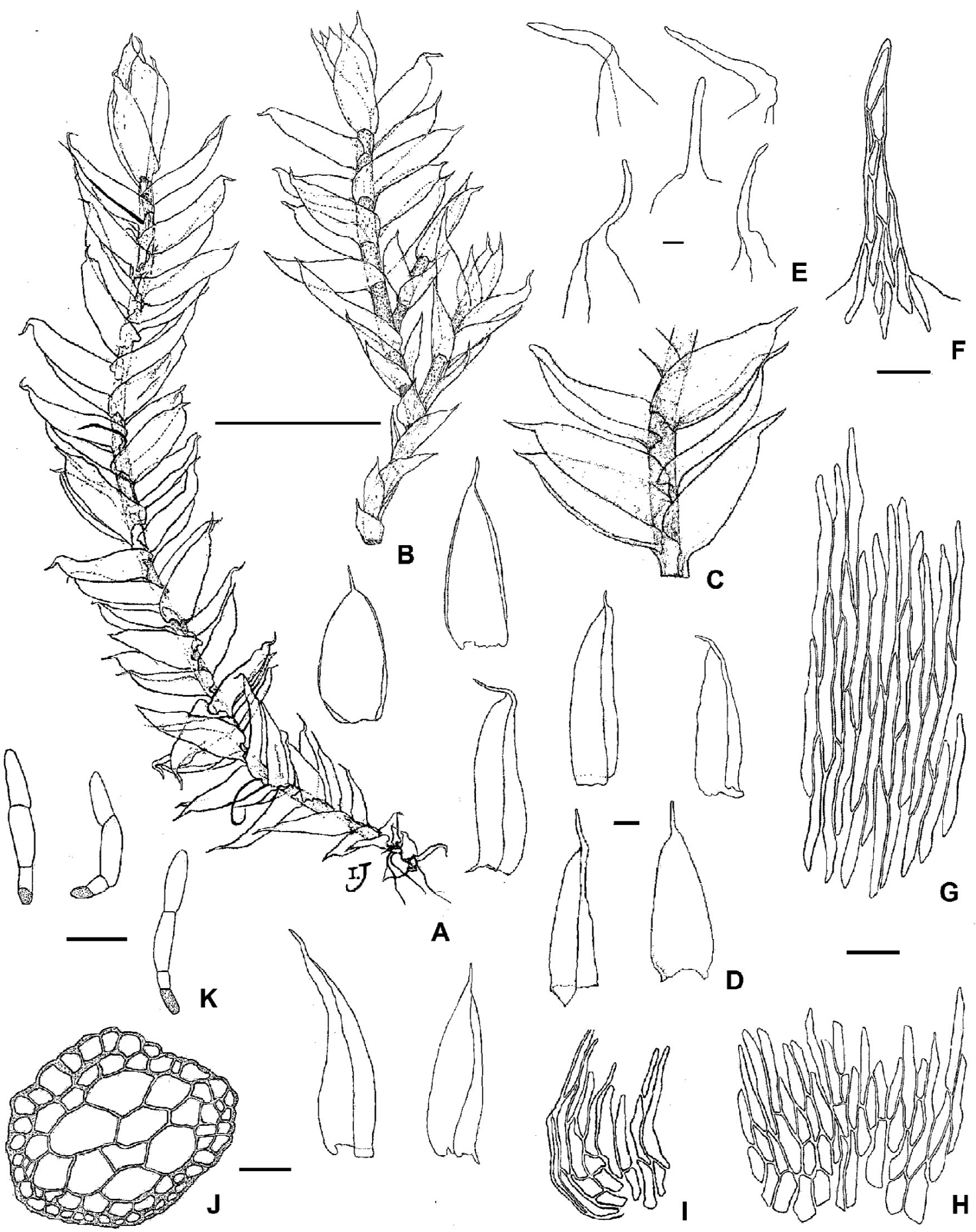

Fig. 1. Catagonium brevicaudatum. A: Hábito de la planta. B-C: Detalles de una rama en húmedo. D. Hojas. E: Detalles del ápice de las hoja vegetativas. F: Células del apículo de la hoja vegetativa. G: Células mediales de la hoja vegetativa. $\mathbf{H}$ : Células basales de la hoja vegetativa. I: Detalle de las células de la aurícula. J: Sección transversal del tallo. K: Pelos axilares. (Escala: A- E: 1 mm, E-K: 25 mm, de G. Suárez 1680 [LIL]). 
por lo que consideramos que este ejemplar fue erróneamente identificado como $C$. nitidum ya que estos caracteres se corresponden con $C$. nitens.

Material examinado. ARGENTINA. Tucumán: Depto. Chicligasta, Parque Nacional Campo de Los Alisos, puesto Los Chorizos, 27¹5'30,8" S; 6553'32,5” W, $1.214 \mathrm{~m}$, epífito sobre corteza, 02/ XI/2012, G. Suárez 1680 (LIL). BOLIVIA. Tarija: Municipio de Padcaya, Canton Emborozu, Reserva Nacional Alarachi, Zona Cayotal, Rio Emborozu Chico, Bosque premontano Tucumano-Boliviano, Musgo sobre tronco de helecho arbóreo (Alsophila), 22014'18" S 64³4'19” W, 1220 m. 21/X/2004. S. Churchill, M. Serrano et al. 23452 (MO).

\section{Contribución de los autores}

GMS coleccionó el material de campo. Ambos autores participaron en la investigación y redacción del manuscrito.

\section{Agradecimientos}

A los revisores por su valiosa contribución en la realización del trabajo y a la Lic. Inés Jaume (Sección Iconografía, FML) por la preparación de la lámina. Este trabajo es financiado por los proyectos PICT 0810 y PIUNT G 631, director de los mismos, GMS.

\section{Bibliografía}

BOWERS, F. D. 1994. Catagoniaceae in A. J. Sharp et al. (editors), Moss Flora of Mexico. Mem. New York Bot. Gard. 69: 972-974.

CHURCHILL, S. P. \&. E. L. LINARES. 1995. Prodromus Bryologiae Novo- Granatensis. Biblioteca "José Gerónimo Triana" 12: 1-924.

COLOTTI, M. T. \& G. M. SUÁREZ. 2017. Novedades sobre la familia Ditrichaceae (Bryophyta) en los pisos superiores de las yungas subtropicales de Argentina. Bol. Soc. Argent. Bot. 52: 277- 289. http://dx.doi.org/10.31055/1851.2372.v52.n2.17443

COLOTTI, M. T., G. M. SUÁREZ \& A. SCHÄFER VERWIMP. 2018. Sobre la presencia de Mittenothamnium reduncum (Hypnaceae) en la Argentina. Bol. Soc. Argent. Bot. 53: 17- 20. http://dx.doi.org/10.31055/1851.2372.v53.n1.19859

FRAHM, J. P. 2003. Manual of tropical bryology. Trop. Bryol. 23: 1-200.

GRADSTEIN, S. R., S. P. CHURCHILL \& N. SALAZAR ALLEN. 2001. Guide to the Bryophytes of Tropical America. Mem. New York Bot. Gard. 86: 1-577.

LIN, S. H. 1984. A taxonomic revisión of Phyllogoniaceae (Brypsida). Part. II. J. Taiwan Mus. 37 (2): 1-54.

MATTERI, C. M. 2003. Los musgos (Bryophyta) de Argentina. Trop. Bryol. 24: 33-100.

SHARP, A. J., H. CRUM \& P. M. ECKEL. 1994. The Moss Flora of Mexico. Mem. New York Bot. Gard. 69: 1- 1113 . 